\title{
Mycobacterium Abscessus: A Rare Cause of Peri-Ductal Mastitis in Endemic Regions
}

\author{
Aisha Shaikh and Lubna Mushtaque Vohra \\ Department of Surgery, The Aga Khan University Hospital, Karachi, Pakistan
}

\begin{abstract}
Mycobacterium abscessus is a rapidly growing non-tuberculous, multi-drug resistant mycobacterium (NTM). Its common clinical presentation includes pulmonary infection followed by wide spectrum of skin and soft tissue infections. Chronic breast conditions, such as peri-ductal mastitis are rarely caused by NTM. Due to an intrinsic and acquired drug resistance to conventional antibiotics and anti-tuberculous therapy, it is often managed with a combination of antibiotics with or without surgical adjuncts. It is important to consider NTM in patients with chronic mastitis who show suboptimal response to initial broad-spectrum antibiotics, and especially when symptoms recur after complete resolution.

This case report describes peri-ductal mastitis caused by mycobacterium abscessus in a 32-year female presenting with a history of painful breast lump and blood stained discharge. With initial diagnosis of nonspecific abscess, she received antibiotic therapy for 4 days at community healthcare setting without promising response. Subsequently, she was diagnosed as a case of peri-ductal mastitis for which quadrantectomy was performed; and surprisingly mycobacterium abscessus was identified on AFB culture. Full recovery was obtained with combination of antibiotics for prolonged period due to frequent relapses.
\end{abstract}

Key Words: Mastitis, Mycobacterium abscessus, Non-tuberculous mycobacteria.

How to cite this article: Shaikh A, Vohra LM. Mycobacterium Abscessus: A Rare Cause of Peri-Ductal Mastitis in Endemic Regions. J Coll Physicians Surg Pak 2020; 30(05):537-540. DOI: https://doi.org/10.29271/jcpsp.2020.05.537.

\section{INTRODUCTION}

Mycobacterium abscessus belongs to a group of rapidly growing environmental mycobacteria found in soil and aqueous environment. ${ }^{1,2}$ It has potential to contaminate medications and medical devices. Contamination of a traumatic or surgical wound or an injection containing contaminated medications may lead to skin and soft tissue infections in an immuno-competent patient. ${ }^{3,4}$ Skin infected with mycobacterium abscessus is usually red, warm, tender to touch and swollen. Additional features may include boils or pus-filled vesicles, fever, chills, and muscle aches. ${ }^{4,6}$

Mastitis caused by such atypical mycobacteria, though reported in literature, is very rare. Clinical presentation may include tender breast lump, sanguineous discharge and chronic abscess formation. ${ }^{1,3,6}$

A definitive diagnosis is based on culture of the organisms from the site of infection or blood, if the infection is severe. ${ }^{1,3}$

Treatment of infections associated with mycobacterium abscessus group is difficult owing to their intrinsic and acquired resistance to the anti-tuberculous drugs and other commonly used antibiotics. Management usually includes use of antimicrobial therapy for variable periods with or without surgery. ${ }^{1-7}$

Correspondence to: Dr. Aisha Shaikh, Department of

Surgery, The Aga Khan University Hospital, Stadium Road,

Karachi, Pakistan

E-mail: aisha.shaikh@aku.edu

Received: February 21, 2020; Revised: April 17, 2020;

Accepted: April 17, 2020

DOI: https://doi.org/10.29271/jcpsp.2020.05.537
This report describes a case of peri-ductal mastitis with abscess formation caused by mycobacterium abscessus in a 32-year female. It was managed surgically with complete resolution of symptoms, followed by recurrence of symptoms after about 2 weeks. Initial bacterial culture and AFB smears were negative; for any microorganism and tissue histology indicated chronic inflammatory reaction with giant cells. Finally, colony formation of mycobacterium abscessus was observed on AFB culture and diagnosis of peri-ductal mastitis secondary to mycobacterium abscessus was established. Patient was treated successfully on combination of antibiotics, but for prolonged period.

To our knowledge, this is the first reported case of peri-ductal mastitis due to mycobacterium abscessus in Pakistan. Details of the case is being shared after taking consent of patient and without breaching individual's confidentiality.

\section{CASE REPORT}

A 32-year non lactating female presented with history of painful lump in her right breast for one month. She had no prior comorbids and was non-smoker. She noticed a painful lump one week after blunt trauma to her right chest.

The skin of the lump was initially red; however, three days later, the nipple started to have a sanguineous discharge. The lump became progressively more painful with the onset of a low grade, intermittent fever without chills. At this point, she was diagnosed as a case of non- specific abscess, for which she was managed initially at community healthcare centre with co-amoxiclave $625 \mathrm{mg} \mathrm{q}$ *12h for 4 days without substantial improvement.

On local examination, a warm, tender, fluctuant lump in the upper inner quadrant of the right breast was found. Overlying skin was 
reddish in color and there was no obvious discharge from the nipple. The right axilla and contralateral breast and axilla were unremarkable.

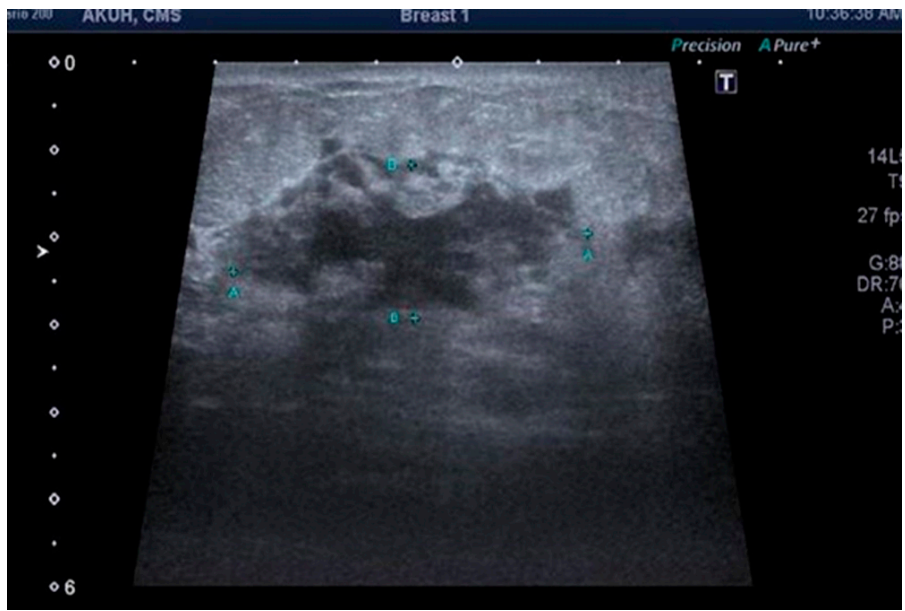

Figure 1a: Irregular heterogeneous area with echoes.

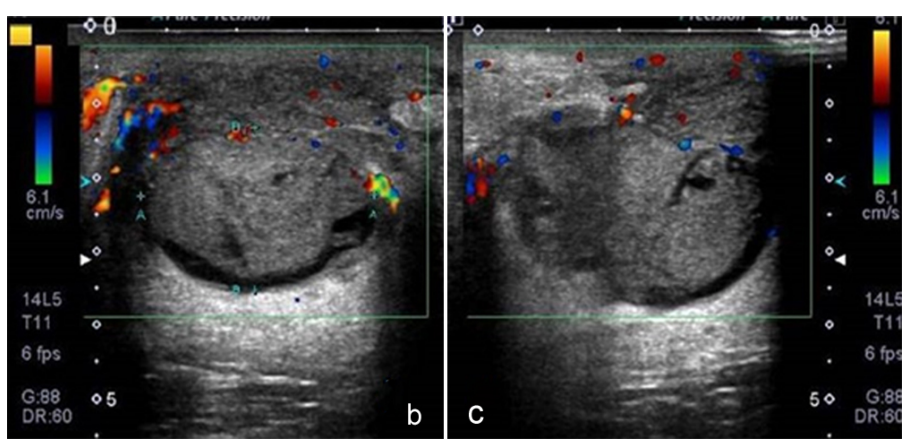

Figure 1b,c: Increased peripheral vascularity.

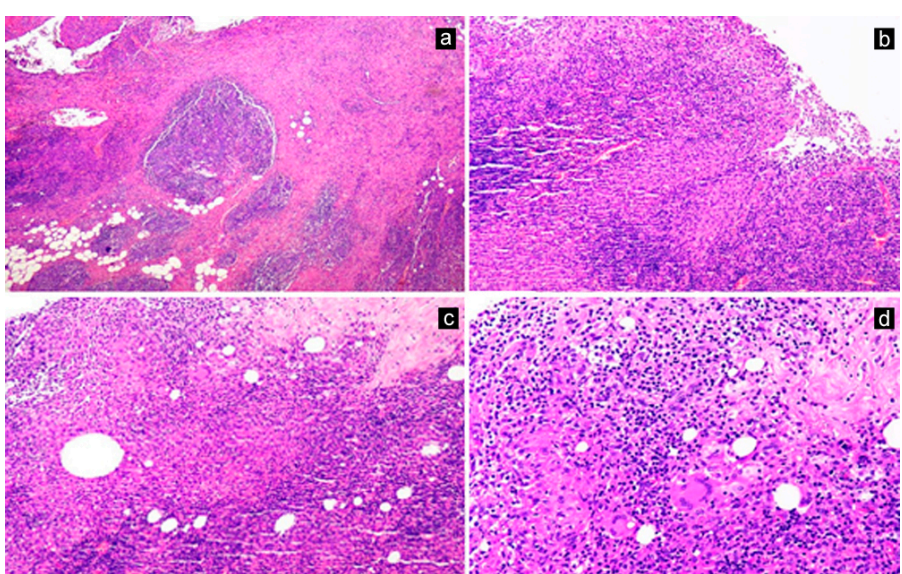

Figure 2: (a) Breast parenchyma with benign mammary ducts. The stroma shows fibrosis and scattered aggregates of inflammatory cells (H\&E; 4X).

(b) Palisading clusters and aggregates of epithelioid histocytes are seen in the stroma (H\&E; 10X).

(c) Collection of epithelioid histiocytes with intererspersed multinucleated giant cells forming granulomat (H\&E; 10X).

(d) Histiocytic collection with scattered multinucleated giant cells (H\&E; 20X).

The complete blood picture showed a marginal increase in leukocytes to $10.8 \times 109 / \mathrm{L}$ (Normal: 7-10.0). An ultrasound identified irregular heterogeneous area extending from 12 O'clock to 4 O'clock position in the right breast measuring $54 \times 45 \times 34 \mathrm{~mm}$, dilated ducts with echoes and mobile echoes (Figure 1a). On color Doppler, there was increased vascularity in periphery of lesion suggesting surrounding tissue reaction (Figure $1 b, c$ ).

A clinical diagnosis of peri-ductal mastitis with abscess in the right breast was followed by a surgical quadrantectomy. Single dose of antibiotic (co-amoxiclave, $1 \mathrm{gm}$ ) was given as prophylaxis. A total of $10 \mathrm{cc}$ pus was removed during the procedure. The wound was left open and daily dressing was advised till first follow-up after a week.

Table I: Important case-related events.

\begin{tabular}{|l|l|l|}
\hline $\begin{array}{l}\text { First week post-surgery } \\
\text { (first follow-up) }\end{array}$ & Normal wound healing & No antibiotics prescribed \\
\hline $\begin{array}{l}\text { Beginning of third week } \\
\text { (second follow-up) }\end{array}$ & $\begin{array}{l}\text { New areas of erythema } \\
\text { and tenderness }\end{array}$ & $\begin{array}{l}\text { Started with clindamycin } \\
\text { (empirically) }\end{array}$ \\
\hline Third week (day 5 of 3rd week) & $\begin{array}{l}\text { Mycobacterium absecuss } \\
\text { discovered on culture }\end{array}$ & $\begin{array}{l}\text { Combination antibiotics as } \\
\text { per C/S reports for 4 weeks }\end{array}$ \\
\hline $\begin{array}{l}\text { Complete wound healing in about 35 days (post-surgery). Antibiotics continued for 4 weeks } \\
\text { (started in 3rd week till 7th week (post-surgery). }\end{array}$ \\
\hline Second relapse & $\begin{array}{l}\text { 3 weeks after wound healing. } \\
\text { - one week off the antibiotics }\end{array}$ \\
\hline $\begin{array}{l}\text { Re-start of combination } \\
\text { antibiotics }\end{array}$ & For 3 months \\
\hline At 4 weeks & $\begin{array}{l}\text { Symptom-free, complete wound healing. Antibiotics } \\
\text { continue. }\end{array}$ \\
\hline
\end{tabular}

The tissue specimens were sent for histological assessment as well as bacterial and AFB cultures. The tissue smears and culture were negative for bacterial growth. Histopathology report demonstrated disruption of some ducts with surrounding mixed inflammatory infiltrate of lymphocytes, plasma cells and neutrophils along with granulomatous reaction and foci of fat necrosis. This provided a definitive diagnosis of peri-ductal mastitis (Figure 2 $a, b, c, d)$

On her first follow-up after a week, wound healing was found satisfactory. During start of third week, however, symptoms resurfaced with new areas of erythema and tenderness. At this time, clindamycin ( $500 \mathrm{mg}$ q*12 hourly) was started empirically. Meanwhile, AFB culture media showed growth of mycobacterium abscessus, with a strong sensitivity profile for amikacin, cefoxitin and clarithomycin, intermediate sensitivity for doxycycline and ciprofloxacin and resistance to trimethoprim /sulfome. Hence, a combination therapy of oral clarithromycin $(500 \mathrm{mg}$ in two divided doses) and intra-venous amikacin (25 mg/kg 3x/wk) was commenced and continued for 4 weeks with a complete wound healing in approximately 35 days.

Three weeks after complete resolution, another new area of erythema and tenderness reappeared. The same antibiotic regimen was re-started for three months and at last follow-up (4 weeks with antibiotics), complete resolution of the erythematous area was witnessed.

Patient will be reviewed after a week, and if evidence of relapse is not found, the oral antibiotics will replace intravenous antibiotic, to be continued for 2-3 months or as decided by clinical judgment in subsequent follow-ups. Table I summarises all important events of wound healing and relapse.

\section{DISCUSSION}

Mycobacterium abscessus and avium complex are most frequently isolated organisms among NTM. ${ }^{1-6}$ Incidence of cases, caused by NTM over the last decade, has markedly increased from 2.7 to 10.2 cases $/ 100,000 .{ }^{1}$ This is especially true for some East Asian regions like Taiwan, where reported incidence is 1.7 cases $/ 100,000$ in comparison to U.S. population, where annual prevalence of $<1 / 10,000$ is reported. ${ }^{2,4,7,8}$ 
As this is the first reported case in Pakistan so nolocal literature is available for comparison; and exact incidence in our setup is not known.

Moore and Frerichs were the first to describe mycobacterium abscessus complex in 1953 as a group of rapidly growing, multidrug-resistant, environmental, NTMs. ${ }^{2}$ Their universal presence in water and soil lead to infections in human organs. ${ }^{2,9,10}$ To date, 3 subspecies of mycobacterium abscessus are known: subsp. abscessus, massiliense, and bolletii. The former two differ from each other by absence of erm gene in abscessus; whereas, infections caused by bolletii are rare.

Although mycobacterium abscessus complex may cause infections involving almost every organ, they are more commonly responsible for a spectrum of skin and soft tissue infections, including surgical site infections (SSI). ${ }^{1-4,7,11,12}$ Disseminated infections and pulmonary infections are mostly reported in immunocompromised hosts, ${ }^{1}$ such as those with an underlying structural lung disease such as cystic fibrosis, bronchiectasis or with prior tuberculosis. $^{2}$

It is rarely implicated in mastitis; and the literature reports only a few cases, that too initially started as skin infection. ${ }^{1-3}$ Clinical presentation of reported cases of mastitis caused by mycobacterium abscessus featured breast swellings, discharge and chronic abscesses that did not respond to broad-spectrum antibiotics. ${ }^{1,3}$ This case had a similar clinical presentation with breast lump; subsequently, ensuing in a nipple discharge which initially responded to the antibiotics only partially.

Infections caused by Mycobacterium abscessus can be acquired in both community and hospital settings.

In the community setting, contaminated water supply systems have been postulated to be the source of human infections which are reported to develop after exposure to certain environmental sources, such as spas and hot springs. ${ }^{2-4}$ Direct contact with contaminated materials/water through traumatic and surgical wounds are other examples.

Mycobacterium abscessus complex outbreaks associated with cosmetic procedures (mesotherapy, tattooing and acupuncture); and other nosocomial transmissions are not uncommon. ${ }^{2,5,6}$

In this case, the patient was not on steroids or immunosuppressive drugs; and had no history of hospitalisation or surgical procedure in recent past. There was a history of a non-penetrating trauma and use of water from water supply system. Though exact mechanism of acquiring infection is difficult to pinpoint in the absence of penetrating injury or open wound; but probable source of infection may be in a community setting related to contaminated water supply system. ${ }^{3}$

Definitive diagnosis of mycobacterium abscessus infection requires isolation of the organism from clinical specimen (percutaneous or excisional biopsy specimens $)^{1-3,5}$ by using various phenotypic and rpo gene-based sequencing methods. ${ }^{2,5}$ In our patient, mycobacterium abscessus growth was determined on AFB culture by phenotypic methods of culture detection on quadrantectomy specimen.
Treatment of mycobacterium abscessus infections entails draining foci of pus or removing the infected tissue with concurrent administration of an appropriate combination of anti-biotics. ${ }^{1,4,7,12}$ Antibiotic sensitivity testing guides appropriate treatment selection for patients..$^{6-7}$ Our patient required surgical management in the form of quadrantectomy, however as the wound was kept open, so patient was not kept on antibiotics in immediate postoperative period. Moreover, mycobacterium abscessus was least suspected at that time. Later on, antibiotics were used as guided by sensitivity profile, which is similar to the sensitivity profile quoted in literature. ${ }^{1,3}$

Various case reports show that despite surgical management and broad spectrum antibiotics, symptoms persisted ${ }^{1,3}$, same was true for our patient who had two relapses post-surgery.

Currently, there is no consensus on optimal antibiotic agents and combination therapy or optimal treatment duration. . $^{1,4,5,7,11,12}$ For established infections, almost all treatment recommendations today include macrolide based (clarithromycin $1000 \mathrm{mg}$ daily or 500 mg divided dose, Azithromycin 250-500 mg daily) combination therapy including parenteral agents such as amikacin ( $25 \mathrm{mg} / \mathrm{kg} 3 \mathrm{x} / \mathrm{wk}$ ), cefoxitin (up to $12 \mathrm{~g} / \mathrm{d}$ in divided doses), tigecycline, imipenem (500 mg 2-4x/wk) or linezolid for atleast 2 weeks to several months, followed by oral anti-microbial therapy. ${ }^{2}$

In our case, with the advent of first relapse, a combination antibiotic therapy (clarithromycin + amikacin) was commenced as guided by sensitivity profile for a period of 4 weeks.

The second relapse led to another prolonged antibiotic course for three-months.

The major constraint of this microbial species is its intrinsic and acquired resistance to most of the currently available antibiotics, classical anti-tuberculous drugs and disinfectants rendering treatment challenging for both clinician as well as patient in terms of cost and compliance. ${ }^{1-7,9-11}$

Preventive methods for reducing the presence of NTM in water supply systems include membrane filtration, hyper chlorination, and maintenance of constant pressure gradients and utilisation of particular pipe materials. ${ }^{2,3}$ Strenous infection control measures may halt the nosocomial transmission and outbreaks in hospital settings. ${ }^{2}$

This case report concludes that rapidly growing NTM need to be considered in differential list in patients with chronic mastitis, who show sub-optimal response to initial antibiotics; and especially, when symptoms recur after complete resolution following surgical management.

\section{PATIENT'S CONSENT:}

Informed consent was taken from the paitent.

\section{CONFLICT OF INTEREST:}

Authors declared no conflict of interest.

\section{AUTHORS' CONTRIBUTION:}

AS: Design of work, literature review and write up for publication. LMV: Conception and design, supervised, critically reviewed and gave final approval. 


\section{REFERENCES}

1. Pasticci MB, Lapalorcia LM, Antonini G, Mencacci A, Mazzolla $R$, Baldelli F. Community-acquired mastitis due to mycobacterium abscessus: A case report. J Med Case Rep 2009; 3:130.

2. Lee MR, Sheng WH, Hung CC, Yu CJ, Lee LN, Hsueh PR. Mycobacterium abscessus complex infections in humans. Emerg infect dis 2015; 21:1638.

3. Yasar KK, Pehlivanoglu F, Sengoz G, Cabioglu N. Successfully treated mycobacterium abscessus mastitis: A rare cause of breast masses. Indian J Med Microbiol 2011; 29:425-7.

4. Lai CC, Tan CK, Chou CH, Hsu HL, Liao CH, Huang YT, et al. Increasing incidence of nontuberculous mycobacteria, Taiwan, 2000-2008. Emerg Infect Dis 2010; 16:294-6.

5. Wongkitisophon P, Rattanakaemakorn P, Tanrattanakorn S, Vachiramon V. Cutaneous mycobacterium abscessus infection associated with mesotherapy injection. Case Rep Dermatol 2011; 3:37-41.

6. Lee SH, Yoo HK, Kim SH, Koh WJ, Kim CK, Park YK, et al. The drug resistance profile of mycobacterium abscessus group strains from Korea. Ann Lab Med 2014; 34:31-7.

7. Benwill JL, Wallace Jr RJ. Mycobacterium abscessus: Challenges in diagnosis and treatment. Current opinion in infect dis 2014; 27:506-10.

8. Nessar R, Cambau E, Reyrat JM, Murray A, Gicquel B. Mycobacterium abscessus: A new antibiotic nightmare. J Antimicrob Chemother 2012; 67:810-8.

9. Wankhade AB, Ghadage D, Bhore AV. Breast abscess due to mycobacterium abscessus: A rare case. Annals of Trop Med and Public Health 2017; 10:447-49.

10. Dickison P, Howard V, O'Kane G, Smith SD. Mycobacterium abscessus infection following penetrations through wetsuits. Australas J Dermatol 2018; 60:57-9.

11. Novosad SA, Beekmann SE, Polgreen PM, Mackey K, Winthrop $\mathrm{KL}$. Treatment of mycobacterium abscessus infection. Emerg Infect Dis 2016; 22:511-4.

12. Gonzalez-Santiago TM, Drage LA. Nontuberculous mycobacteria: Skin and soft tissue infections. Dermatolo clin 2015; 33:563-77. 